\title{
Quantum Optic Entanglement Using Graphene Clad Surface Plasmonic Polariton Device
}

Partha Pratim Sahu ( $\nabla$ ppstezu@gmail.com )

Tezpur University School of Engineering

\section{Research Article}

Keywords: graphene-clad, surface plasmonics, entanglement

Posted Date: May 17th, 2021

DOl: https://doi.org/10.21203/rs.3.rs-493890/v1

License: (c) (i) This work is licensed under a Creative Commons Attribution 4.0 International License. Read Full License 


\title{
Quantum optic entanglement using Graphene clad surface plasmonic polariton device \\ Partha Pratim Sahu \\ Department of Electronics and Communication Engg, Tezpur University, Napaam, Tezpur-784028, Assam, India \\ Email: ppstezu@gmail.com
}

\begin{abstract}
:
Here, Graphene clad plasmonics waveguide is introduced as a two surface plasmonic polariton modes interference (GTSPPMI) coupler to obtain optically manipulated quantum interference. The manipulation of Handel $\mathrm{Ou}$ Handel (HOM) quantum interference is demonstrated theoretically in nano-scale two modes coupler through refractive index modulation in Graphene clad with incidence of an ultra fast optical pulse energy. The quantum entanglement of fidelity $\sim 97.5 \%$ is obtained by incidence of optical pulse of energy $5.12 \mathrm{pJ}$ and width $3.8 \mathrm{ps}$ in Graphene cladding. Our results promise to obtain fast and compact optical reconfiguring of quantum plasmonics circuit in comparison to electrooptic and themooptic coupler.
\end{abstract}

Keywords: graphene-clad, surface plasmonics, entanglement

\section{Introduction}

Recently, quantum optic technology has been focused for high speed information processing [1]-[2], secure communication [3]-[4] and instrumentation [5]-[6], due to having its unique quantum mechanical properties such as superposition, entanglement etc. Moreover, it also provides high noise margin, easy operation and low power requirement [7]. The use of bulk optics encounters the problems of its precision against the vibration and susceptibility to external electromagnetic radiation. To deal with these restrictions, integrated optics has already been employed with its advantages such as low signal loss, light weight, reliable operation, precision phase control [8]-[9]. But integrated optics technology is also restricted with fundamental property of diffraction of light energy to reduce device size for large scale integration. Here, surface plasmonic polariton (SPP) wave has a capability to overcome this restriction through its light-electron excitation at metal-dielectric interface providing localization of electromagnetic energy at nano-scale dimension [10]-[11]. The control of electromagnetic energy within nano scale using SPP wave promises large scale integration of quantum circuit. The SPP waveguide has large metallic loses due to use of metal- dielectric interface and other disadvantages such as temporal and spectral distortion are arisen due to modal and chromatic dispersion in SPP waveguide. The modal dispersion can be reduced by allowing fewer number of SPP modes propagated in the waveguide. For reduction of metallic losses, new materials having small value of extinction constant of refractive index and also free electrons (as in metal) should be chosen. In this direction, Dirac plasmons have already been demonstrated experimentally through the coupling with infrared light [12]-[13] at graphene -dielectric interface due to having excellent electronic and photonic property. In comparison to other of 2D materials such as transition metal chalcogenides, black phosphorus, hexagonal boron nitride, Graphene shows excellent nonlinear absorption over UV, visible and infrared wavelength due to having its zero band gap [14]. The graphene plasmonics have already been used in integrated waveguide due to its positive value of imaginary part of refractive index [15]- [16]. Controlled quantum gates are key devices in quantum system requiring manipulation of quantum states. The manipulation of quantum entanglement has already been performed either through electro-optic [17] or thermo-optic control [18]-[20] on wave propagation inside waveguide. The thermooptic and electro-optic reconfiguration of quantum states restrict the speed of quantum processing due to having low response time and less precision in phase control. Moreover, occurrence of unwanted thermooptic effect in unstable regions of waveguide provides phase error. Recently, the modulation of refractive index in GaAsInP cladding with incidence of optical pulse energy (E) of 15-27 pJ and pulse width of 100-280 ps has already been reported for optical pulse controlled propagation of SPP modes reducing the problem of phase error [21]-[22]. For further reduction of phase error, it is required to decrease optical pulse energy and pulse width for accurate manipulation of quantum entanglement in the quantum devices. In this direction, graphene provides the modulation of refractive index with incidence of ultra fast infrared light pulse through nonlinear absorption [23].

Here, a two surface plasmonics polariton mode Graphene clad-silicon waveguide has been presented for accurate manipulation of quantum entangled states using ultrafast infrared optical pulse. The HOM depth of quantum interference has been formulated as a function of optical pulse energy incident on graphene cladding region. Due to having low optical pulse energy and pulse width, the manipulation becomes faster than that of thermooptic and electro-optic quantum device. 


\section{Device concept}

Figure-1a shows a $2 \times 2$ Graphene clad two SPP mode interference (GTSPPMI) quantum device consisting two modes coupling region of width $2 \mathrm{w}$ and thickness $\mathrm{t}$, two single mode input access and two output access waveguides of width $\mathrm{w}$ and thickness $\mathrm{t}$. The waveguide has silicon core surrounded by graphene cladding. The complex refractive index of graphene is represented as $\mathrm{n}_{2}=\mathrm{n}+\mathrm{jk}$ (where $\mathrm{n}=$ real part of refractive index and $\mathrm{k}=$ imaginary part of refractive index ) whereas refractive index of silicon core is denoted as $\mathrm{n}_{1}$. The real part of refractive index of graphene shows nonlinear refractive index change $(\Delta n(E))$ with incidence of optical pulse of energy $E$ and width $T_{P}$. The $\Delta n(E)$ is written as [24][25]

$$
\Delta n(E)=n(E)-n(0)=\frac{n_{n l} E}{1.065 A_{C} T_{P}}
$$

where, $n_{n l}=$ nonlinear refractive index coefficient of graphene nwith incidence infrared light $=1.5 \times 10^{-9}(\mathrm{~cm})^{2} \mathrm{~W}^{-1}[24]$ and $A_{c}=$ area of left and right side graphene cladding on which optical pulse energy is incident for optical manipulation of quantum states $=2 \mathrm{w}_{\mathrm{c}} \mathrm{t}, \mathrm{w}_{\mathrm{c}}=$ width of left or right side graphene cladding. To obtain quantum surface plasmonics phenomena in graphene clad TMI device, the spontaneous parametric down converted wavelength of $0.810 \mu \mathrm{m}$ is incident on input access waveguides and quantum mechanically represented by $\left|a_{1}\right\rangle$ and $\left|a_{2}\right\rangle$. When it interacts with graphene-silicon interface, the local electric fields are induced at the interface and represented as surface plasmonic plariton (SPP) modes depending on the width (w) of silicon core. The propagation constants of SPP modes are written as

$$
\beta_{m}\left(n_{1}, n_{2}(0)\right)=\beta_{m}^{r}\left(n_{1}, n_{2}(0)\right)+j \beta_{m}^{\text {im }}\left(n_{1}, n_{2}(0)\right)
$$

where, $\mathrm{m}=0,1,2 \ldots$ represents fundamental, first order, second order... respectively. The $\beta_{m}^{r}\left(n_{1}, n_{2}(0)\right)$ and $\beta_{m}^{i m}\left(n_{1}, n_{2}(0)\right)$ are real and imaginary part of propagation constant without application of optical pulse. The real part of effective refractive index $n_{m, e f f}^{r}\left(n_{1}, n_{2}(0)\right)$ is determined by using effective index method (where the continuity condition is obtained at the graphene-silicon interface [13]-[14]) for $\lambda=0.810 \mu \mathrm{m}, \mathrm{n}_{1}=3.69, \mathrm{n}_{2}(0)=2.879+\mathrm{j} 1.564$, and $\mathrm{t}$ $=2 \mu \mathrm{m}$. Figure-2(a) shows that first and second order modes start appearing at $\mathrm{w}=0.18$ and $0.27 \mu \mathrm{m}$ respectively. For two modes coupling, we have chosen width $\mathrm{W}_{\mathrm{TMI}}=2 \mathrm{~W}=0.18 \mu \mathrm{m}$, (where $\mathrm{W}_{\mathrm{TMI}}=$ width of two modes coupling region). The imaginary part of propagation constant of excited modes in TSPPMI region provides propagation length of modes written as $L_{P, m}=\frac{1}{2 \beta_{m}^{i m}\left(n_{1}, n_{2}(0)\right)}$. The beat length $\left(L_{\pi}\right)$ is coupling length to obtain phase difference of $\pi$ between two excited modes and determined by using formula written as $L_{\pi}=\frac{\pi}{\beta_{0}^{r}\left(n_{1}, n_{2}(0)\right)-\beta_{1}^{r}\left(n_{1}, n_{2}(0)\right)}$, where $\beta_{0}^{r}\left(n_{1}, n_{2}(0)\right)$ and $\beta_{1}^{r}\left(n_{1}, n_{2}(0)\right)$ are real part of propagation constants of fundamental and first order mode respectively. The $L_{\pi}$ and $L_{P, m}$ as function of TMI core thickness $\mathrm{t}$ are represented in Figure 2 (b). The propagation lengths of the excited SPP modes increase with $\mathrm{t}$ and are more than $L_{\pi}$ at $\mathrm{t} \geq 2 \mu \mathrm{m}$. The $L_{\pi}$ remains almost constant with $t$ as propagation characteristics are independent t.. For coupling of fundamental and first order modes, we have chosen $\mathrm{t}=2 \mu \mathrm{m}$ in which $L_{\pi}$ is obtained as $\sim 0.745 \mu \mathrm{m}$.

To control the propagation of excited modes in TMI region, optical pulse of energy $\mathrm{E}$ and wavelength $\lambda=0.81 \mu \mathrm{m}$ is incident on left and right graphene cladding region and the propagation constant of SPP modes generated in TSPPMI region are modulated as

$$
\beta_{m}\left(n_{1}, n_{2}(E)\right)=\beta_{m}^{r}\left(n_{1}, n_{2}(E)\right)+j \beta_{m}^{i m}\left(n_{1}, n_{2}(E)\right)
$$

where, $\beta_{m}^{r}\left(n_{1}, n_{2}(E)\right)$ and $\beta_{m}^{i m}\left(n_{1}, n_{2}(E)\right)$ are represented as real part and imaginary part of propagation constant respectively, modulated with optical pulse energy E. The transformation matrix representing input to output quantum state transition is represented as, 


$$
\begin{aligned}
& T_{\text {matrix }}=\left(\begin{array}{ll}
T_{11} & T_{12} \\
T_{21} & T_{22}
\end{array}\right)= \\
& \left(\begin{array}{ll}
\cos (\Delta \theta(E) / 2) e^{-\left(\beta_{0}^{i m}+\beta_{1}^{i m}\right) L / 2} & j \sin (\Delta \theta(E) / 2) e^{-\left(\beta_{0}^{i m}+\beta_{1}^{i m}\right) L / 2} \\
j \sin (\Delta \theta(E) / 2) e^{-\left(\beta_{0}^{i m}+\beta_{1}^{i m}\right) L / 2} & \cos (\Delta \theta(E) / 2) e^{-\left(\beta_{0}^{i m}+\beta_{1}^{i m}\right) L / 2}
\end{array}\right)
\end{aligned}
$$

where $\Delta \theta(E)=$ phase difference between fundamental and first order mode $=\Delta \phi(0)+\Delta \phi(E)$

$$
\begin{aligned}
& \Delta \phi(0)=\left(\beta_{0}^{r}\left(n_{1}, n_{2}(0)\right)-\beta_{1}^{r}\left(n_{1}, n_{2}(0)\right)\right) \mathrm{L} \\
& \Delta \phi(E)=\frac{2 \pi}{\lambda}\left(\Delta n_{1, e f f}^{r}\left(n_{1}, n_{2}(E)\right)-\Delta n_{01, e f f}^{r}\left(n_{1}, n_{2}(E)\right)\right) L=\text { phase change obtained by incidence of optical }
\end{aligned}
$$

pulse of energy $E$ incident on left and right graphene cladding region

$$
\begin{aligned}
& \Delta n_{0, e f f}^{r}\left(n_{1}, n_{2}(E)\right)=n_{0, e f f}^{r}\left(n_{1}, n_{2}(E)\right)-n_{0, e f f}^{r}\left(n_{1}, n_{2}(0)\right) \\
& \Delta n_{1, e f f}^{r}\left(n_{1}, n_{2}(E)\right)=n_{1, e f f}^{r}\left(n_{1}, n_{2}(E)\right)-n_{1, e f f}^{r}\left(n_{1}, n_{2}(0)\right)
\end{aligned}
$$

The $n_{0, \text { eff }}^{r}\left(n_{1}, n_{2}(E)\right)$ and $n_{1, \text { eff }}^{r}\left(n_{1}, n_{2}(E)\right)$ are real part of effective refractive index of fundamental and first order modes with incidence of optical pulse energy respectively. Figure -2(c) shows $\Delta \phi(E)$ versus $\mathrm{E}$ for left and right graphene cladding area, $\mathrm{A}_{\mathrm{C}}=1,2$ and $3 \mu \mathrm{m}^{2}$ with $2 \mathrm{w}=0.18 \mu \mathrm{m}, \mathrm{n}_{1}=3.69, \mathrm{n}_{2}(0)=2.879+\mathrm{j} 1.564$, and $\mathrm{t}=2 \mu \mathrm{m}$. As $\mathrm{A}_{\mathrm{C}}$ decreases, optical pulse energy coupled to graphene cladding for the manipulation of propagation characteristics reduces. Optical pulse energy to obtain $\Delta \phi(E)$ of $0.5 \pi$ between fundamental and first order modes is obtained as $5.12 \mathrm{pJ}, 20 \mathrm{pJ}$ and $42 \mathrm{pJ}$ for $\mathrm{A}_{\mathrm{C}}=1,2$ and $3 \mu \mathrm{m}^{2}$ respectively. The fiber to device coupling efficiency also depends on $\mathrm{A}_{\mathrm{C}}$ and is determined by using $\eta=\frac{A_{C}}{\pi r^{2}}\left[n_{1}^{2}-n^{2}(E)\right]$. The $\eta$ increases with the increase of $\mathrm{A}_{\mathrm{C}}$ as shown in Figure-3b. For $\mathrm{A}_{\mathrm{C}}=$ $1 \mu \mathrm{m}^{2}$, optimized values of $\eta$ is estimated as $\sim 93 \%$ where diameter of fiber $=2 \mathrm{r}=2.4 \mu \mathrm{m}$ and $\mathrm{n}(\mathrm{E})=3.069$ and that of $\mathrm{E}$ to obtain $0.5 \pi$ phase change is determined as $5.12 \mathrm{pJ}$.

\section{Quantum entanglement}

The manipulated quantum entanglement is made through optical pulse controlled coupling of two SPP modes generated by incidence of single photon at each of inputs $a_{1}$ and $a_{2}$. The output quantum entangled states $\left|b_{1}(E)\right\rangle$ and $\left|b_{2}(E)\right\rangle$ at the output $b_{1}$ and $b_{2}$ are written as

$$
\begin{aligned}
& \left|b_{1}(E)\right\rangle=\left[\cos (\Delta \theta(E) / 2)\left|a_{1}\right\rangle+\sin (\Delta \theta(E) / 2)\left|a_{2}\right\rangle\right] e^{-\left(\beta_{0}^{i m}+\beta_{1}^{i m}\right) L / 2} \\
& \left|b_{2}(E)\right\rangle=\left[\cos (\Delta \theta(E) / 2)\left|a_{2}\right\rangle+\sin (\Delta \theta(E) / 2)\left|a_{1}\right\rangle\right] e^{-\left(\beta_{0}^{i m}+\beta_{1}^{i m}\right) L / 2}
\end{aligned}
$$

Here, the manipulation of quantum entanglement states is considered for cross coupling TMI device $(\Delta \phi(0)=\pi)$. The coincidence count $(\mathrm{CC})$ of the device is written as

$$
\begin{aligned}
& N_{C}(E)=K\left|T_{11} T_{22}+T_{12} T_{21}\right|^{2} \\
& \quad=K\left[1-\frac{1}{2} \sin ^{2}(\Delta \phi(E))-\frac{1}{2} \sin ^{2}(\Delta \phi(E)) \psi\right]
\end{aligned}
$$


where, $\mathrm{K}=$ constant estimated from incident number of photons, $\psi=$ overlap integral representing distinguishability of two surface polarization polariton [26]-[28] $=\exp \left(-\Delta \omega^{2} t_{c}^{2} / 8\right) \exp \left(-\frac{2 \tau^{2}}{t_{c}^{2}}\right), \Delta \omega=$ difference between two central frequencies of Gausian spectral distribution, $\tau=\Delta \mathrm{x} / \mathrm{c}=$ time lag between the arrivals of two photons at the ports of TMI coupler and $\mathrm{t}_{\mathrm{c}}$ $=$ coherence time corresponding to coherence length $1_{c}=$ c.t. . Figure $-4 \mathrm{a}$ shows $\mathrm{CC}$ versus $\Delta \mathrm{x}$ determined by using the equation (6) for incident optical pulse energy $\mathrm{E}=0,2.13,3.33$ and $5.12 \mathrm{pJ}$ with pulse width of $3.8 \mathrm{ps}$. The HOM dip is quantified with quantum visibility which is obtained by using formula, $\mathrm{V}(\mathrm{E})=\frac{N_{\infty}(E)-N_{o}(E)}{N_{\infty}(E)}$, (where $N_{\infty}(\mathrm{E})=$ coincidence count for classically distinguishable photons $(\tau \rightarrow \infty)$ and $N_{o}(\mathrm{E})=$ coincidence count for quantum mechanically indistinguishable photons $(\tau \rightarrow 0)$ ). At $\mathrm{E}=0$, approximately zero HOM dip is obtained due to having fully cross coupling of quantum fields whereas maximum quantum visibility of 0.95 is obtained with incidence of optical pulse energy of $\mathrm{E} \sim 5.12$ $\mathrm{pJ}$ due to having phase change $\Delta \phi(E)=0.5 \pi$. The quality of quantum entanglement is quantified in terms of quantum fidelity $\mathrm{F}$ which is written as

1

$$
\mathrm{F}(\mathrm{E})=(1+\mathrm{V}(\mathrm{E})) / 2
$$

Figure $-4 b$ shows $F(E)$ versus $E$ determined by using the equation (7). Quantum fidelity is maximum at $E=5.12 \mathrm{pJ}$ due to having phase change of $\Delta \phi(E)=0.5 \pi$. By varying incident optical pulse energy, quantum fidelity can be modulated. Quantum entanglement also depends on spatial degree of coherence $(\gamma)$ of SPP modes (which corresponds to photons)as visibility is directly proportional to $\gamma$ [29]. As roughness of graphene-dielectric interface [29], the $\gamma$ has negligible effect on quantum entanglement due to having constant value of unity. By considering bending loss of $0.12 \mathrm{~dB}$ [30], radius of curvature of bending $(\mathrm{R})$ and separation between access waveguides $\left(2 \mathrm{H}_{\mathrm{T}}\right)$ are estimated as $8 \mu \mathrm{m}$ and $10 \mu \mathrm{m}$ respectively and longitudinal length of access waveguide is determined as [30] $\mathrm{L}_{\mathrm{T}}=\sqrt{H_{T}\left(4 R-H_{T}\right)} \sim 11.6 \mu \mathrm{m}$. The overall length of the device is estimated as $\mathrm{L}_{\mathrm{ToT}}=2 \mathrm{~L}_{\mathrm{T}}+\mathrm{L}_{\pi}=23.945 \mu \mathrm{m}$ which is $\sim 167$ times and 545 times smaller than that of thermooptically manipulated TMI [30] and electrooptically manipulated Mach Zender [17] quantum device respectively.

\section{Conclusion}

Here, optical pulse controlled GTSPPMI device is presented and designed as a basic component for reconfigurable integrated quantum plasmonics device. The manipulation of quantum entanglement is performed by incidenting very low energy optical pulse in graphene clad. High fidelity of $97.5 \%$ is obtained at optical pulse energy of $5.12 \mathrm{pJ}$ with two mode SPP modes coupler of width of $180 \mathrm{~nm}$. The length of GTSPPMI device is 167 times and 545 times smaller than that of both thermooptically manipulated TMI [30] and electrooptically manipulated Mach Zender quantum device [17] respectively.

\section{References}

1. T. D. Ladd, F. Jelezko, R. Laflamme, Y. Nakamura, C. Monroe and J. L. O’Brien. 2013, Nature, Vol- 464, pp 45-47.

2.P.Walther, 2005, Nature Vol-434, pp169-173..

3. Ursin R., F. Tiefenbacher,T. Schmitt-Manderbach and A. Zeilinger 2007, Nature Phys, Vol-3, pp 481-483.

4. I. Marcikle, H.deRiedmatten, W. Tittel, H. Zbinden and N. Gisin, 2003, Nature, Vol-421, pp 509-511.

5. T. Ono, R. Okamato and S. Takeuchi,2013, Nature Communication, Vol-4, pp2426-2434.

6. P P Sahu,2018," Proc of International Conference on Fiber Optics and Photonics (invited), 12- 15 December 20.

7. J. L. O’Brien, A. Furusawa and J. Vuckovic, 2009, Nature Photonics, vol-3, pp687-695, 2009.

8. P P. Sahu, 2009, IEEE J. Sel. Top. Quantum. Elect.,Vol-15, pp1537-1541. 2009.

9. P. G. Kwiat, 2008, Nature Vol-453, pp294- 295, 2008.

10. J. A. Schuller, E. S. Barnard, W. Cai, Y. C. Jun, J. S. White and M. L. Brongersma, 2010 „. Nature Materials, Vol- 9, pp $193-204$.

11. P. Berini and I. De Leon, 2011, Nature Photonicsm Vol- 6, pp16-24.

12. A. N. Grigorenko, M. Polini and K. S. Novoselov, 2012, Nature Photonics, Vol-6, pp749 -758.

13. Fei Z. et.al.,2011, Nano letters, Vol-11, pp 4701-4705.

14.J.W. You, S.R. Bongu, Q. Bao and N.C. Panoiu, 2019, Nanophotonics, Vol 8(1), pp 63-97.

15. J. T. Kim, and S. Y. Choi, 2011, Optics Express, Vol-19, pp 24557-24562.

16. J. W. Weber, V. E. Calado and M. C. M van de Sanden, 2010, 2010, Applied Physics Letters, Vol-97, pp091904.

17. K. H. Luo, S. Brauner, C. Eigener, P. R. Sharapova, R. Ricken, T. Meier, H, /Herrmann and C. Silberhorn, 2019, Science Advances, Vol-5, eaat 1451. 
18. Mathews J. C. F., Politi A., Sefenov and O'Brien J. L., 2009, Nature Photonics, Vol-3, pp8-11.

19. Shadbolt et.al. 2012, Nature phtotonics, Vol-6, pp45-49..

20. P P Sahu, 2019, Results of physics, Vol-12, pp1329-1333,.

21. P. P. Sahu, 2016, J of lightwave techno., Vol-34, pp1300-1305..

22. P. P. Sahu, 2012, Appl. Opt., Vol. 51, pp. 2601-2605.

23. H. Zhang, S. Virally, Q Bao, L, K, Ping, S. Massar, N. Godbout and P. Kockkaert, 2012, Optics Letter, Vol-37, pp1856-1858, 2012.

24. E. Henry, P. J. Hale, J. Moger, A. K. Savchenkoand M. A. Mikhailov, 2010. Physics Rev. Lett, Vol-105, pp097402.

25. R. S. Grant and W. Sibbett, 1991, Applied. Phys. Lett., Vol. 58, pp. 1119-1121.

26. P. P. Sahu, 2019, Plasmonics, Vol-14, 875-879.

27. H. Weinfurter, A. Zeilinger and M.Zukoowski, 1995, Applied physics B:Lasers and optics, Vol-60, S111- S117 .

28. S. A. Guebrou, J. Laverdant, C. Symonds, S. Vignoli, and J. Bellessa, 2012,, Optics letter, Vol. 37, pp $2130-2132$.

29. D. Y,1 Jung, S. Y. Yang, H. Park, W. C. Shin, J. G. Oh, B. J. Cho and S.Y. Choi, 2015, Nano Convergence Vol.2(11), pp1-17.

30. P. P. Sahu,", 2018, Quantum Information Processing, Vol-17, pp150-155. 


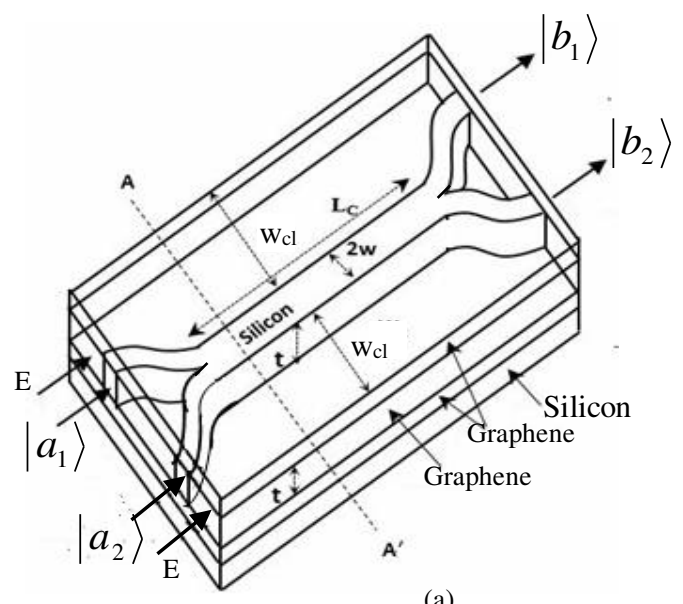

(a)

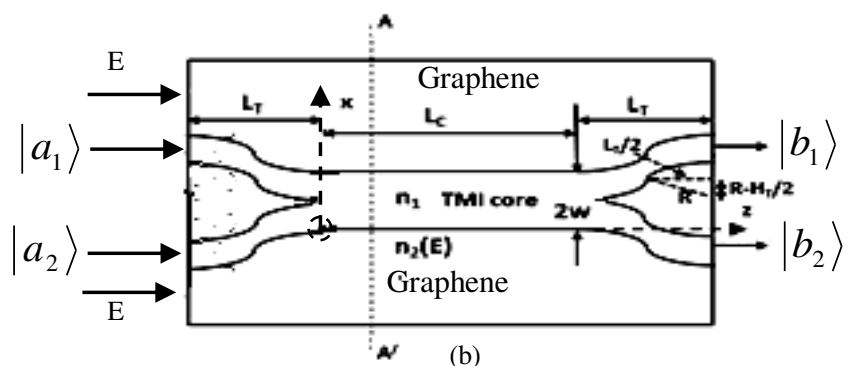

(b)

A

\begin{tabular}{|l|l|l|}
\hline \multicolumn{2}{|c|}{ Graphene } \\
\hline Graphene & sticon & Graphene \\
\hline & Sticonsuastant \\
\hline Scl & Graphene \\
\hline
\end{tabular}

(c)

Fig-1 (a) 2x2 two mode interference (TMI) coupler of TMI core width $2 \mathrm{w}$ and core thickness $\mathrm{t}$ with graphene cladding (b) 2D TMI coupling device with incident of optical pulse energy $\mathrm{E}$ in left and right graphene region (c) cross section view of TMI region of the device. 

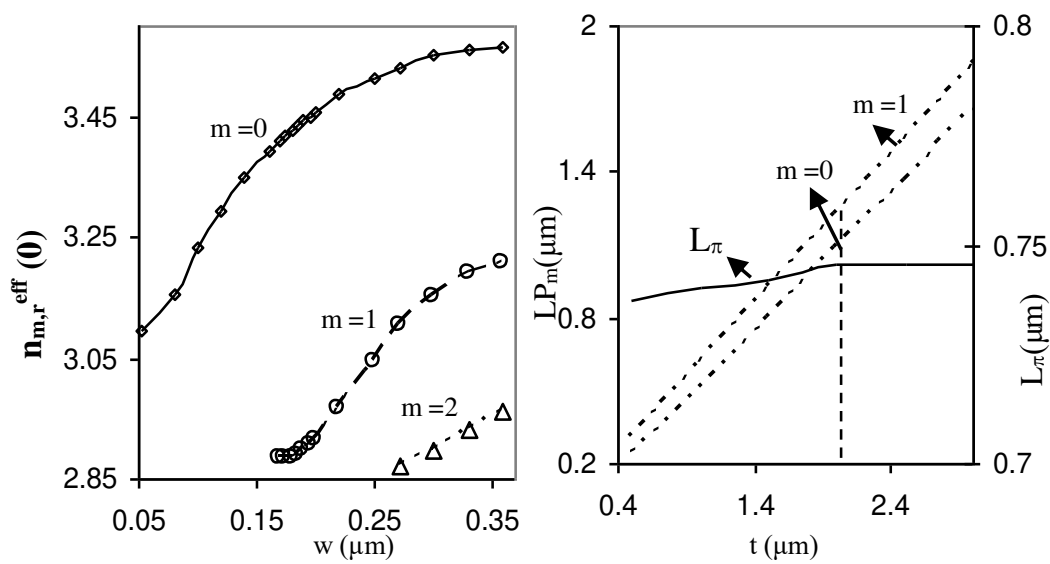

Fig-2: (a) $\mathbf{n}_{\mathbf{m}, \mathbf{r}}$ eff $(\mathbf{0})$ versus $\mathrm{w}$ (where $\mathrm{m}=0,1,2$ represents fundamental , first order and second order mode and optical pulse energy $E=0 \mathrm{pJ}$ ) estimated by using the equation (2)-(3) with $\lambda=0.810 \mu \mathrm{m}, \mathrm{n}_{1}=3.69, \mathrm{n}_{2}(0)=$ $2.879+\mathrm{j} 1.564$ and $\mathrm{t}=2 \mu \mathrm{m}$. Only two modes (fundamental and first order ) are excited and propagated for $0.265 \mu \mathrm{m}>\mathrm{w}>0.175 \mu \mathrm{m}$ (b) Dependence of propagation length $(L P m$, where $m=0,1)$ and coupling length $\left(L_{\pi}\right)$ on $t$ for $2 \mathrm{w}=0.18 \mu \mathrm{m}$. The coupling length remains almost independent on $\mathrm{t}$ but for $\mathrm{t}>1.9 \mu \mathrm{m}$, the $\mathrm{L}_{\pi}$ remains less than that of propagation lengths of both excited modes.
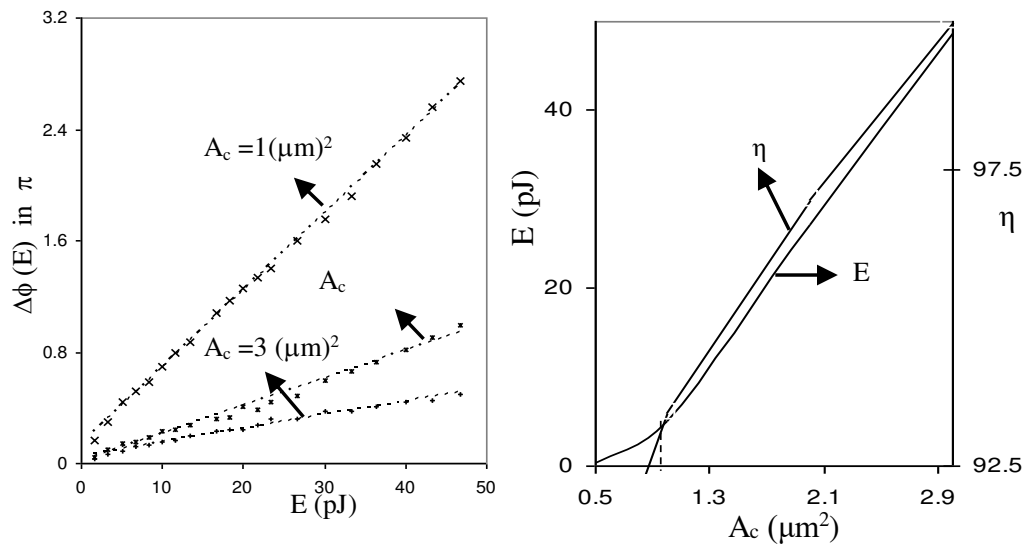

Fig-3: (a) $\Delta \phi$ (E) versus $E$ estimated by using dispersion equation equations for $\mathrm{A}_{\mathrm{c}}=1,2,3(\mu \mathrm{m})^{2}$ for $\lambda=0.810 \mu \mathrm{m}, \mathrm{n}_{1}=3.69, \mathrm{n}_{2}(0)=2.879+\mathrm{j} 1.564,2 \mathrm{w}$ $=0.18 \mu \mathrm{m}$ and $\mathrm{t}=2 \mu \mathrm{m}$. Phase change $\Delta \phi(\mathrm{E})$ due to incident of optical pulse increases linearly with pulse energy $\mathrm{E}$. The rate increase of $\Delta \phi(\mathrm{E}) \mathrm{w}$ $\mathrm{r}$ to $\mathrm{E}, \frac{d(\Delta \phi(E))}{d E}$ for $\mathrm{A}_{\mathrm{c}}=1,2$ and $3(\mu \mathrm{m})^{2}$ are $0.0554,0.0203$ and $0.0097(\pi / \mathrm{pJ})$ respectively. The value of $\frac{d(\Delta \phi(E))}{d E}$ decreases with $\mathrm{A}_{\mathrm{c}}$ due to increase of incident area. (b) Dependence of $\mathrm{E}$ and fiber to device coupling efficency on $A_{C}$. The optimized value of $E$ and $\eta$ are obtained as $\sim 5.12 \mathrm{pJ}$ and $93 \%$ respectively at $\mathrm{A}_{\mathrm{C}}=1(\mu \mathrm{m})^{2}$ 

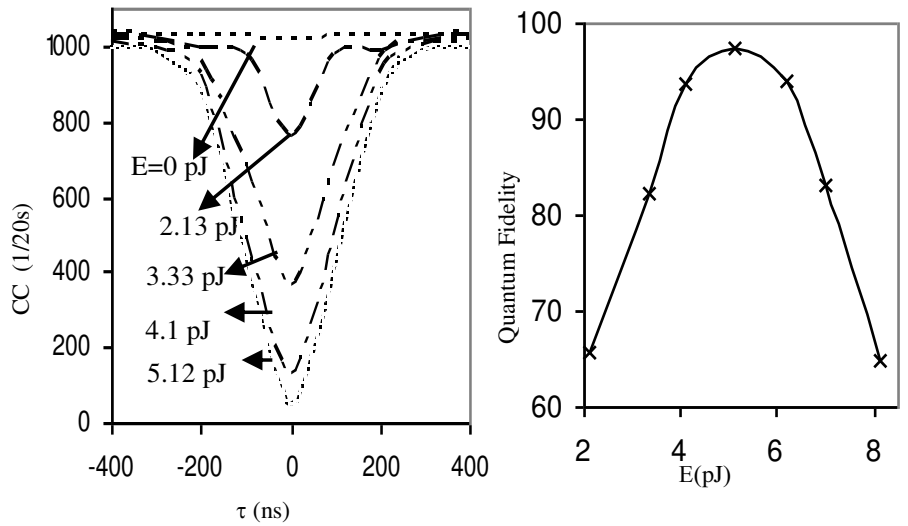

Fig-4: (a) Coincidence count (CC) versus $\Delta \mathrm{x}$ (where $\Delta \mathrm{x}=\mathrm{c} . \tau, \tau=$ time lag between the arrivals of two photons at the ports of TMI coupler) manipulated theoretically with optical pulse energy $\mathrm{E}=0$, $2.13,3.33,4.12$ and $5.12 \mathrm{pJ}$ and estimated by using the equation (2)-(3) with $\Delta \omega=0.986 \times 10^{12} \mathrm{~s}^{-1}, \lambda=0.810 \mu \mathrm{m}, \mathrm{n}_{1}=3.69, \mathrm{n}_{2}(0)=$ $2.879+\mathrm{j} 1.564,2 \mathrm{w}=0.18 \mu \mathrm{m}$ and $\mathrm{t}=2 \mu \mathrm{m}$. Quantum visibility for $\mathrm{E}=2.13,3.33,4.12$ and $5.12 \mathrm{pJ}$ is determined theoretically by using equation $\mathrm{V}(\mathrm{E})=\frac{N_{\infty}(E)-N_{o}(E)}{N_{\infty}(E)}$, (where $N_{\infty}(\mathrm{E})=$ coincidence count for classically distinguishable photons $(\tau \rightarrow \infty)$ and $N_{o}(\mathrm{E})=$ coincidence count for quantum mechanically indistinguishable photons $(\tau \rightarrow 0)$ ). as $\sim 0.317,0.645,0.873$ and 0.95 respectively (b) Quantum fidelity versus E manipulated with optical pulse incident in left and right cladding of TMI device. 


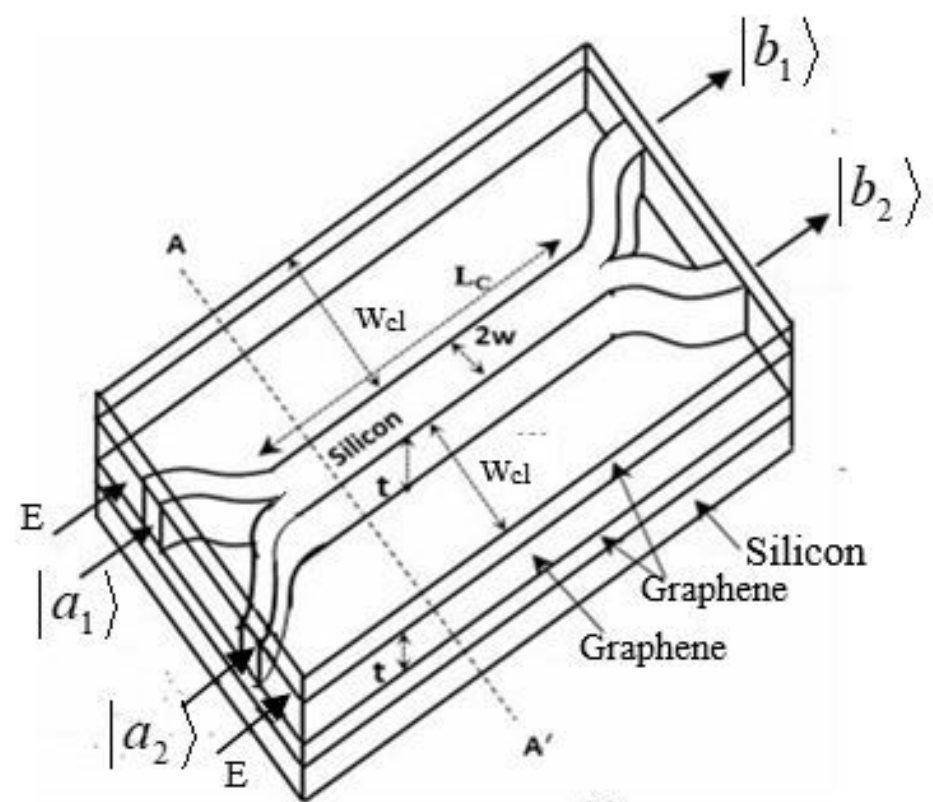

(a)

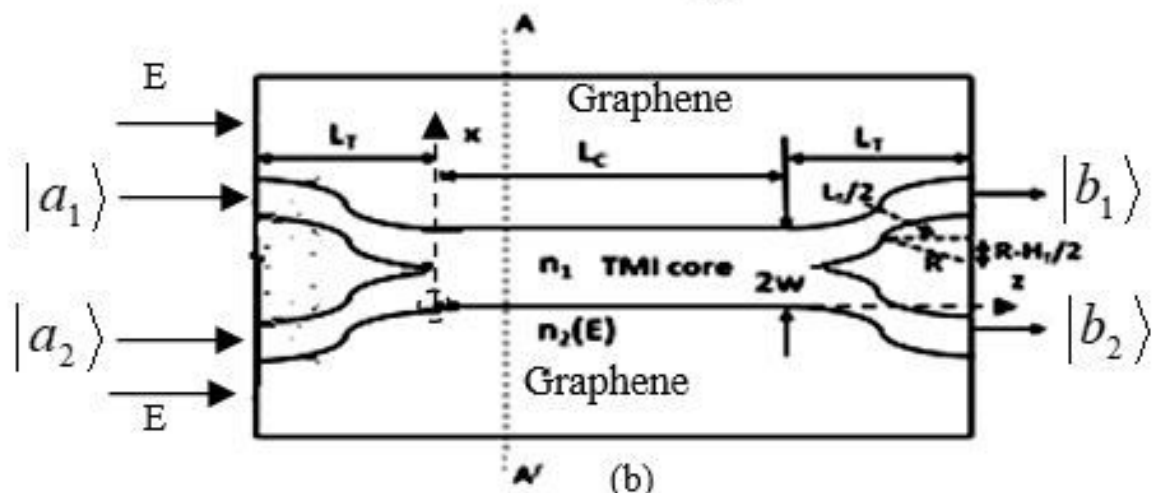

(b)

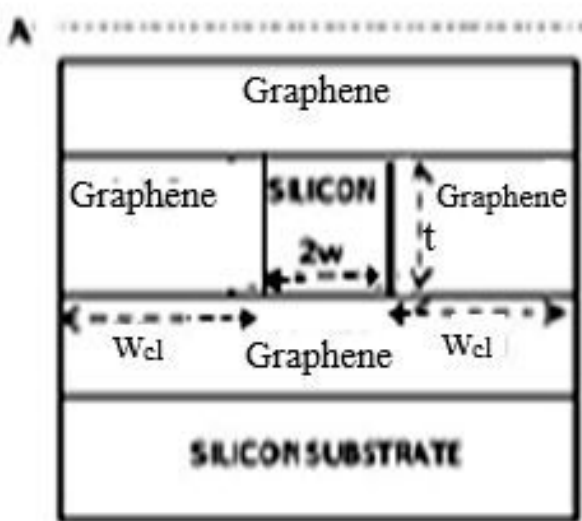

(c)

\section{Figure 1}

(a) $2 \times 2$ two mode interference (TMI) coupler of TMI core width $2 \mathrm{w}$ and core thickness $t$ with graphene cladding (b) 2D TMI coupling device with incident of optical pulse energy $E$ in left and right graphene region (c) cross section view of TMI region of the device. 

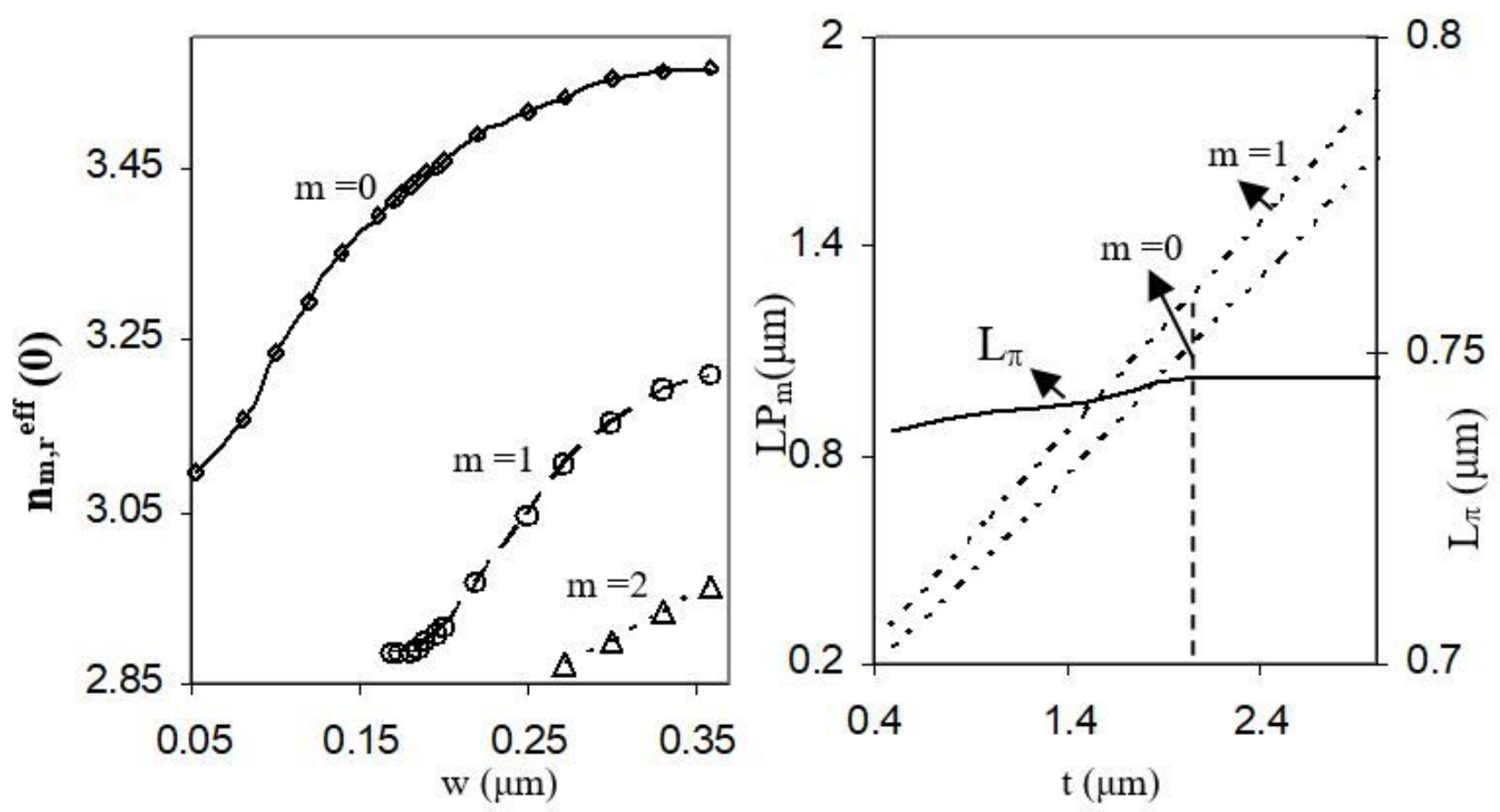

Figure 2

please see the manuscript file for the full caption
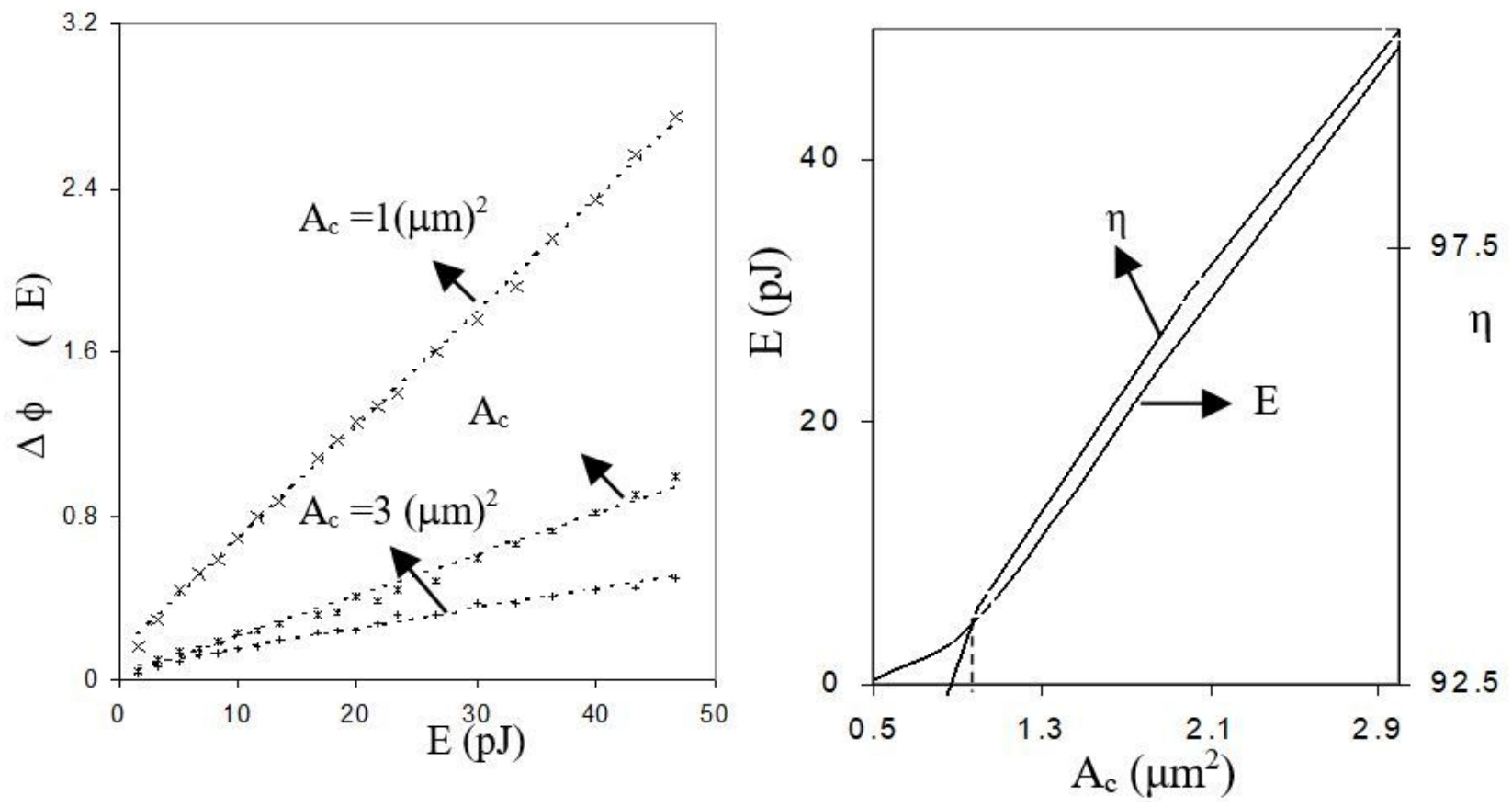

Figure 3

please see the manuscript file for the full caption 

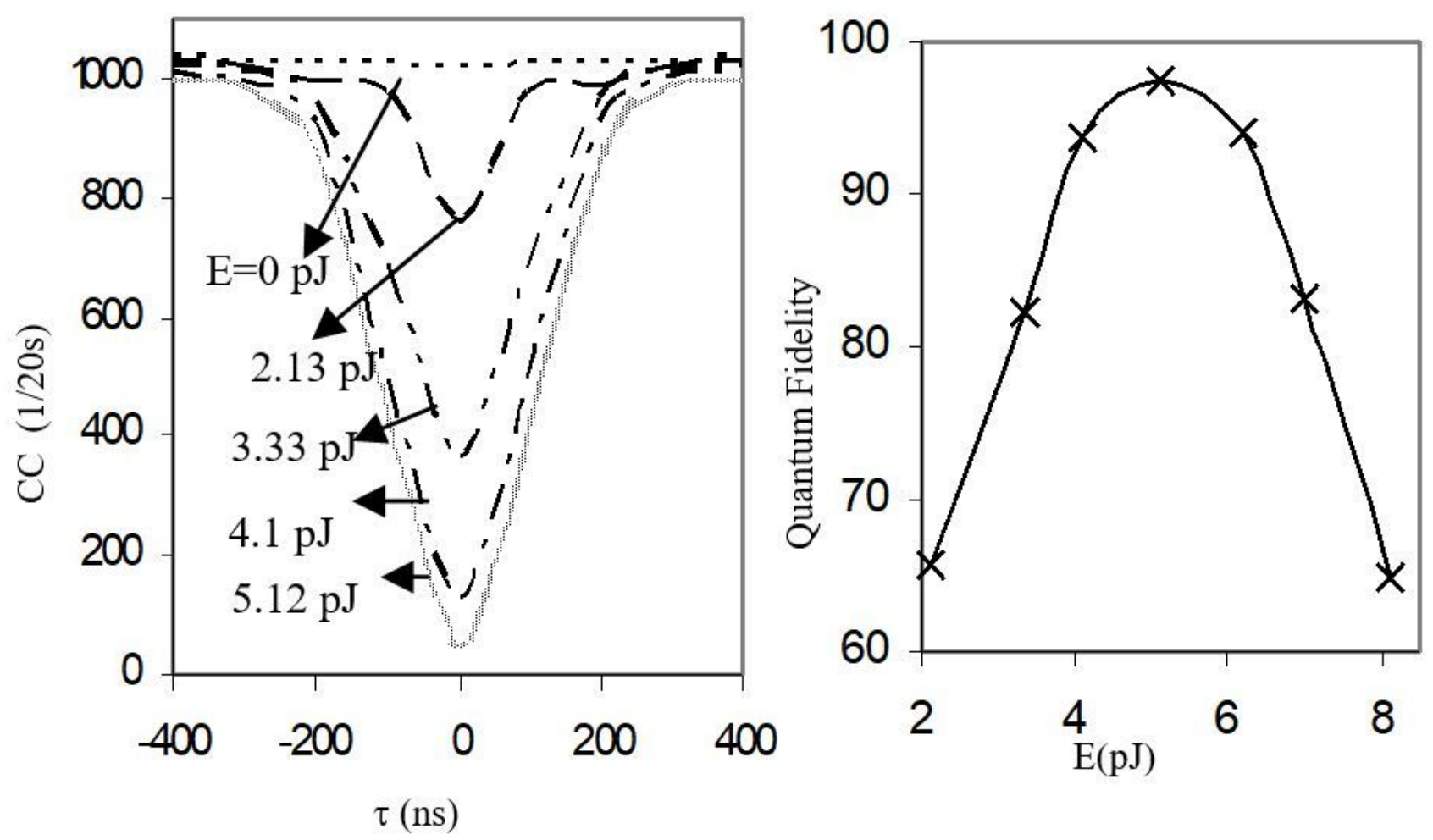

Figure 4

please see the manuscript file for the full caption 